\title{
The role of the hospital pharmacy in the storage and supply of antidotes
}

\author{
Luisa Lombardo, ${ }^{1}$ Salvatore Di Rosa, ${ }^{2}$ Fabio Venturella ${ }^{3}$ \\ ${ }^{1}$ Ospedale San Giovanni di Dio, Agrigento; ${ }^{2}$ AO Ospedali Riuniti Villa Sofia-Cervello, Palermo; ${ }^{3}$ Dipartimento di Scienze per \\ la Promozione della Salute G. D’Alessandro, Università di Palermo, Italy
}

\begin{abstract}
Cases of poisoning have been analyzed with the aim of estimating both the incidence and the seriousness of such xenobiotic effects, the major determinant agents, and the related antidotes used in hospital departments. From 2009 to November 2012 , a survey of the availability of antidotes in the emergency room services was carried out in all hospital pharmacies under the Palermo Health Authority: Policlinico Paolo Giaccone, ARNAS Civico di Cristina Benefratelli, Azienda Ospedaliera Ospedali Riuniti Villa Sofia-Cervello. Figures show that the majority of poisoning events were caused by the use/abuse of drugs, especially psychopharmaceuticals, alcohol and narcotic substances. This overdose of medical drugs and its various causes is a cause for concern and highlights the fact that this tendency has not regressed over the years. Doctors and pharmacists should raise patients' awareness about how to use drugs correctly, and make adequate information available to all patients in order to, first, reduce the risks, and second, reduce the cost of treatment for intoxication.
\end{abstract}

\section{Introduction}

Intoxications represent an important phenomenon among developed countries. The significant diffusion of chemical products, such as products for cleaning the house and garden, and medical drugs in particular, makes the risk of intoxication very high, especially when safety precautions are not observed during use. ${ }^{1}$ The epidemiology of acute intoxication and poisoning is poorly represented in national and international medical literature. Information of an acceptable quality can be traced through hospital admissions data. Information can be voluntary or involuntary (e.g. overdose of medical drugs). In order to avoid an over-

Correspondence: Luisa Lombardo, Ospedale San Giovanni di Dio, contrada Consolida, 92100 Agrigento, Italy.

Tel. +39.320 .7685390 .

E-mail: iviava@hotmail.it

Key words: therapeutic index, overdose, poison, availability, supply, pharmacy.

Received for publication: 11 December 2012.

Revision received: 27 March 2013.

Accepted for publication: 10 April 2013.

This work is licensed under a Creative Commons Attribution NonCommercial 3.0 License (CC BY-NC 3.0).

(C) Copyright L. Lombardo et al., 2013

Licensee PAGEPress, Italy

Italian Journal of Medicine 2013; 7:259-264

doi:10.4081/itjm.2013.259 dose, it is very important to refer to the therapeutic index (TI). This is the ratio given by lethal dose divided by effective dose: $\mathrm{TI}=\mathrm{LD}_{50} / \mathrm{ED}_{50} . \mathrm{LD}_{50}$ is the dose that, if given to a statistically significant number of animals, leads to the death of $50 \%$ of the population. $\mathrm{ED}_{50}$ is the dose that, if given to a statistically significant number of animals, determines a standardized positivity effect of the drug on $50 \%$ of the population. Every substance that harms the organism can be considered a poison, but what helps in the identification of a toxic substance is the dose that provokes the harmful effects. As Paracelsus writes: The dose makes the poison. Poison is an organic or inorganic, vegetable, animal, synthetic or extractive, simple or complex, soluble or non-soluble substance that if introduced into the organism provokes illness or death through a chemical or biochemical mechanism. Poisoning can be due to many different causes: drugs, personal hygiene products, domestic cleaning products, insecticides and garden products, fuels, and industrial and agricultural products. ${ }^{2}$ Poisoning can also be caused by an overdose. The absolute overdose consists in the administration of an excessive dose of a drug by mistake or with suicide/homicidal intent. Major or minor risk depends on the TI. A relative or false overdose occurs when the drug dose administrated is included in the therapeutic range but with an increase in its plasma concentrations. This can be caused by: hepatic pathologies, renal pathologies, hypoalbuminemia, drug interactions.

The assistance needed by a person with symptoms of poisoning may differ according to the poison, the way it entered the body, and whether the person is conscious or unconscious. A substance that can counteract 
the action of a poison is called an antidote. The hospital pharmacy plays an important role both in providing and storing antidotes (sometimes they are not found in Italy or are only available as galenic formulations). Through a specific or non-specific mechanism, they improve the prognosis quad vitam or quad functionem of the intoxication. Some criteria must be respected in ensuring sufficient stocks of antidotes are stored and available such as: proven efficacy, urgency of use, duration of treatment, any adverse effects, availability, cost. $^{3}$ The aim of this paper is to analyze the most relevant acute intoxication and poisoning cases in a 3year period on a sample of the main hospitals in Palermo. The objective is that of estimating the incidence and seriousness of such xenobiotic actions, the major causative agents, as well as the relative antidotes used in the hospital departments through the data on antidote use of hospital pharmacies of the hospitals taking part in the study. Such an analysis is also aimed at establishing the correct procedure for prioritizing antidotes and to verify any reduction in the number of cases over the years as a result of an increase in available information and prevention. (Antidote priorities, up-dated in 2012, are available in the online Appendix, Tables A1-A4). Given that, for some type of intoxications, the immediate availability of antidotes is absolutely essential to provide correct treatment, it is very important that the emergency room (ER) has adequate antidote availability. This paper aims to verify the real availability of such antidotes in the various hospitals examined, and to check that pharmacists and doctors give adequate instructions on how to avoid intoxication from incorrect drug use.

\section{Methods of research}

A survey on the actual availability of antidotes in the emergency room services in hospitals under the Palermo Local Health Authority was carried out between 2011 and January-November 2012. Results were compared with the previous 2-year period in order to establish whether there had been a possible increase or decrease in antidote use. Percentages of antidote use were obtained from all hospital pharmacies of the main Palermo hospitals: Policlinico Paolo Giaccone, ARNAS Civico di Cristina Benefratelli, Ospedali Riuniti Villa Sofia-Cervello, and by analyzing printed and computer files regarding all patient access to the emergency room services of these hospitals. For the purpose of this study, every drug or chemical substance used to treat intoxication from poisoning, primarily to counteract the toxic effect, has been considered an antidote. Antidote management is controlled by an accounts system that regulates sorting, supply and storage of antidotes to the various departments. This accounts system is different in each hos- pital. Symptomatic drugs are not included in the antidotes. According to this study, the majority of acute intoxications are due to drugs.

The objective was to underline that the drug percentages do not reflect the real situation because, since the creation of detox centers, most people who have any kind of drug abuse problem goes to these centers rather than to ERs. In terms of toxic exposure, the most frequent is orally $(85.3 \%)$, confirming the prevalence of episodes of intoxication from drugs and alcohol; these are followed by inhalation $(5.9 \%)$, ocular $(3.7 \%)$, intravenous $(2.9 \%)$ and skin $(2.2 \%)$. This study also analyses the supply of antidote stocks in order to provide information about the type and quantity of antidotes on an Italian national, regional and provincial basis available to all the national healthcare system hospitals (considering especially the more expensive antidotes and those less used). The Pavia Poison Control Center (PCC) provided a dedicated online database on its website (http://www.cavpavia.it). This National Antidote Database (Banca dati Nazionale degli Antidoti, $\mathrm{BaNdA}$ ) reports all the available antidotes in each national healthcare structure that collaborates with the project and shares its data. This both allows an antidote that is urgently needed for a patient to be found and optimizes drug supply. ${ }^{5}$ General results about the use of antidotes both in the emergency room services and Emergency Medicine will be taken into account together with data from the other departments. The survey was carried out in the main hospitals of Palermo, Sicily. In particular, antidote use at the Policlinico Paolo Giaccone is shown in Figure 1.

The presence of each antidote was verified both in the ER and in the other departments. The percentage of antidotes indicated in Figure 1 is evaluated on the cases of intoxications obtained through consulting data from the Emergency Room Register and information from the hospital pharmacy.

The study particularly focused on the antidotes used in ER, as these are the antidotes most requested from the hospital pharmacy. The analysis of ER supply was performed on the entire list of antidotes. It appears that vials of calcium gluconate $10 \%(25.12 \%)$ and atropine sulfate $(22.59 \%)$ are those most used. Figure 2 shows the number of intoxications registered in the Emergency Department records at the ARNAS Civico di Cristina Benefratelli hospital. The most used antidotes in this hospital are shown in Figure 3. From these data we can conclude that most intoxication episodes are caused by drugs and illegal drugs, with some other particular intoxications such as pesticides (accidental consumption caused by wrong labeling or contact through agricultural use) requiring the use of atropine.

Also the characteristics related to the Ospedali Riuniti Villa Sofia-Cervello (Figure 4) show more poisoning episodes due to medicines and alcohol, leading 
to the request for antidotes as shown in Figure 5 . Among the poisoning episodes studied in this hospital, attention should be drawn to those caused by accidental ingestion of toxic plants, like the Mandrake plant that was wrongly considered edible; this required the use of physostigmin. It should be noted that the high percentage of requests to the hospital pharmacy for naxolone is due not to its use as an antidote in ER, but as an emergency antidote in the operating room, where the use of morphine is expected. The use of ethanol as an antidote is linked to the consumption of low cost products containing methanol that do not satisfy legal requirements. To deal with this kind of poisoning, fomepizole would be more suitable. This is a more specific and efficient antidote in the prevention of metabolic acidosis but unfortunately, due to its high cost, it is not found in any hospital pharmacy under the Palermo Local Health Authority.

\section{Discussion and Conclusions}

The principal role of the hospital pharmacy is to provide medicines and drugs (sometimes those less common ones) and it is obviously the best source of information concerning pharmacology and pharma-

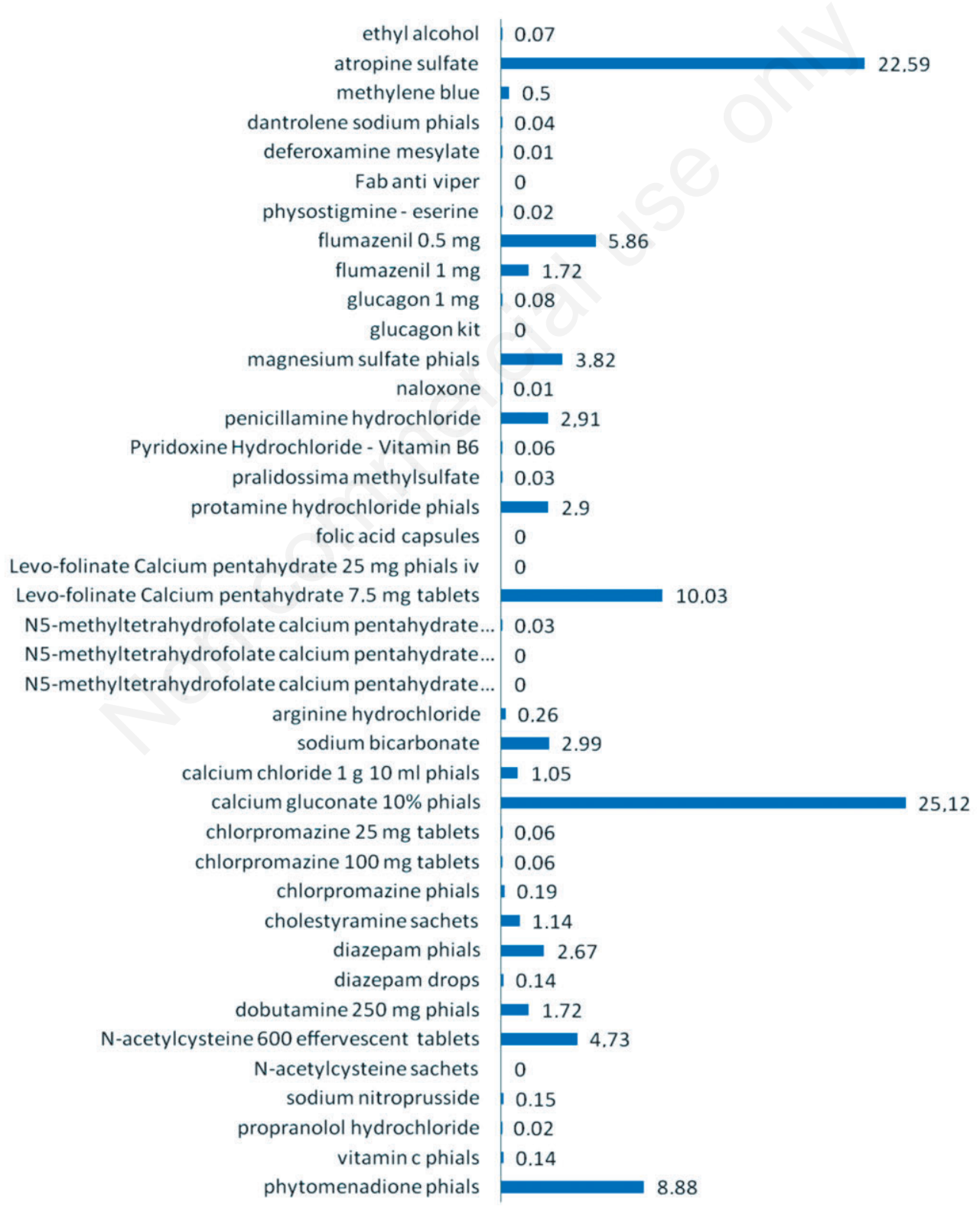

Figure 1. Percentage of antidotes used from 2009 to November 2012 at the Policlinico Paolo Giaccone, Palermo. Commercial trade names have been replaced by active ingredients. 
ceuticals. Without a doubt, the hospital pharmacy plays a fundamental role in providing antidotes and responding to the need for urgent treatment. Some antidotes cannot easily be found either because they are only available in galenic formulation (e.g. physostigmin) or because they are not available on the market in Italy in an appropriate form (e.g. hydroxocobalamin, sodium thiosulfate, Prussian blue).

The results of this study show a slight increase in the number of cases of intoxication throughout the pe- riod 2009-2012. Figure 6 summarizes the total number of cases recorded from analyzing the register of the Emergency Departments of the three hospitals involved in the study. This shows a tendency for the number of cases to remain almost the same in the first 2-year period and shows a slight increase in the last year. Given that most cases concern pharmaceutical intoxication and drug abuse, it can be concluded that more information from health experts is needed.

The evaluation of antidote use showed there are

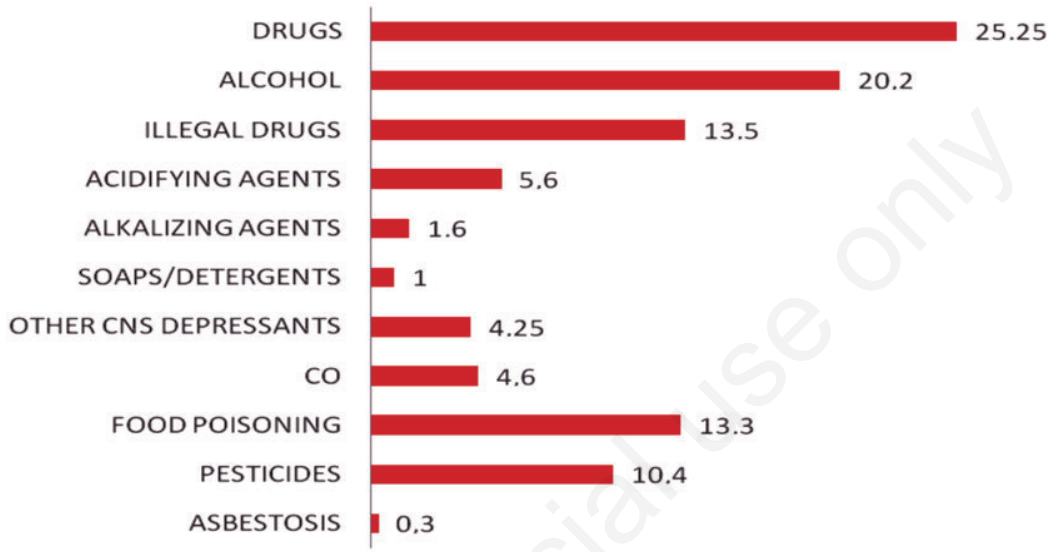

Figure 2. Episodes of poisoning from 2009 to November 2012 at the ARNAS Civico di Cristina Benefratelli hospital, Palermo.

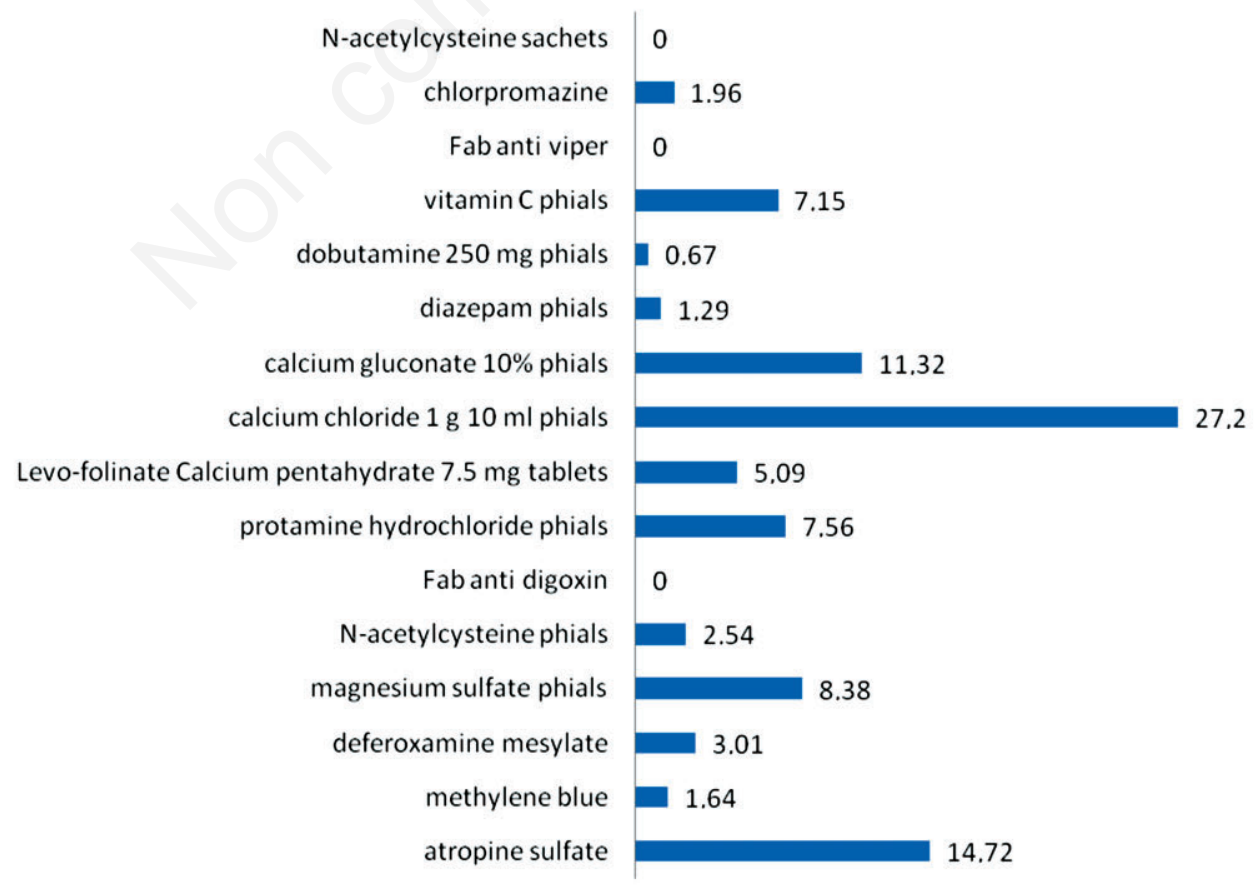

Figure 3. Percentage of antidotes used from 2009 to November 2012 at the ARNAS Civico di Cristina Benefratelli hospital, Palermo. 


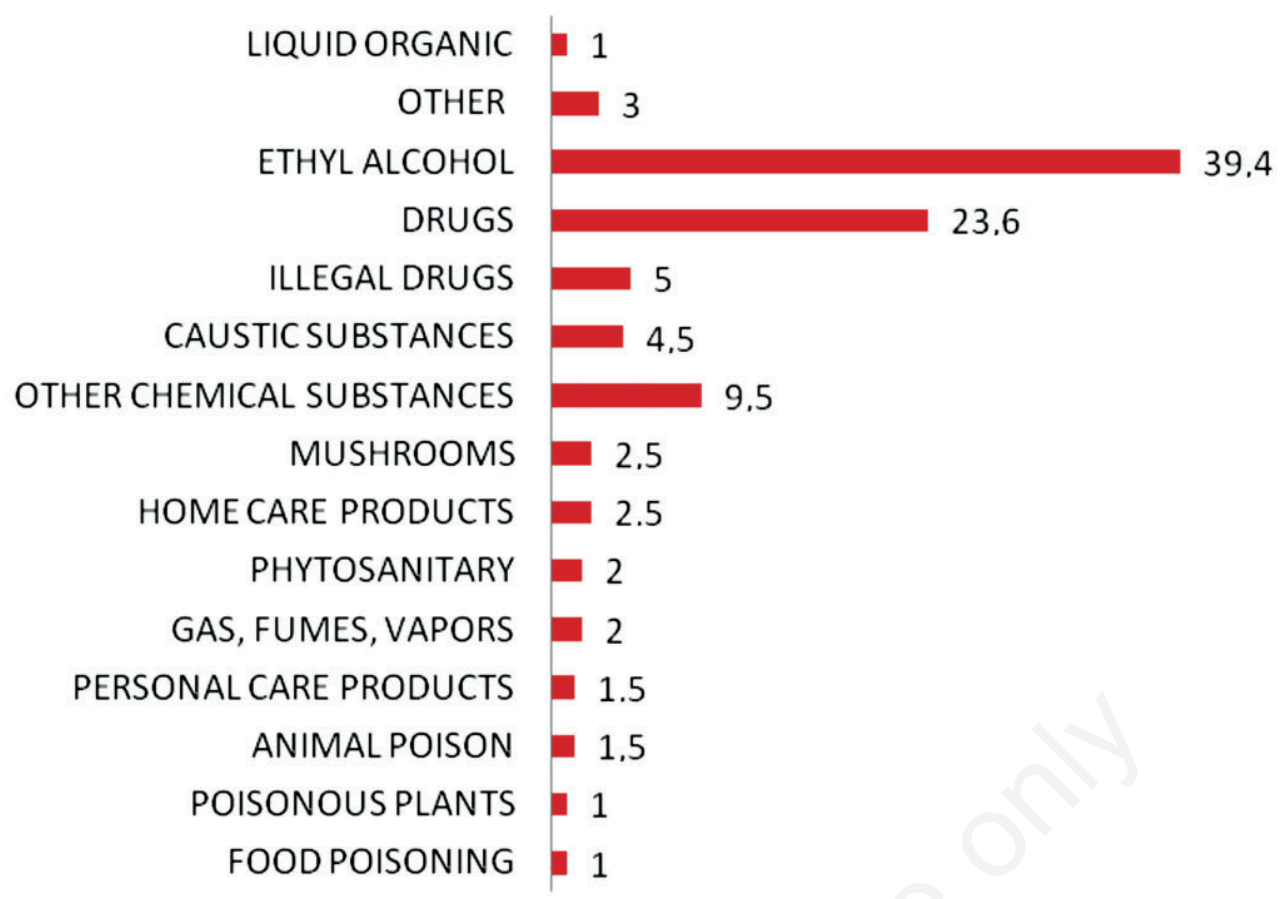

Figure 4. Percentage of poisonings from 2009 to November 2012 at the Ospedali Riuniti Villa Sofia-Cervello, Palermo.

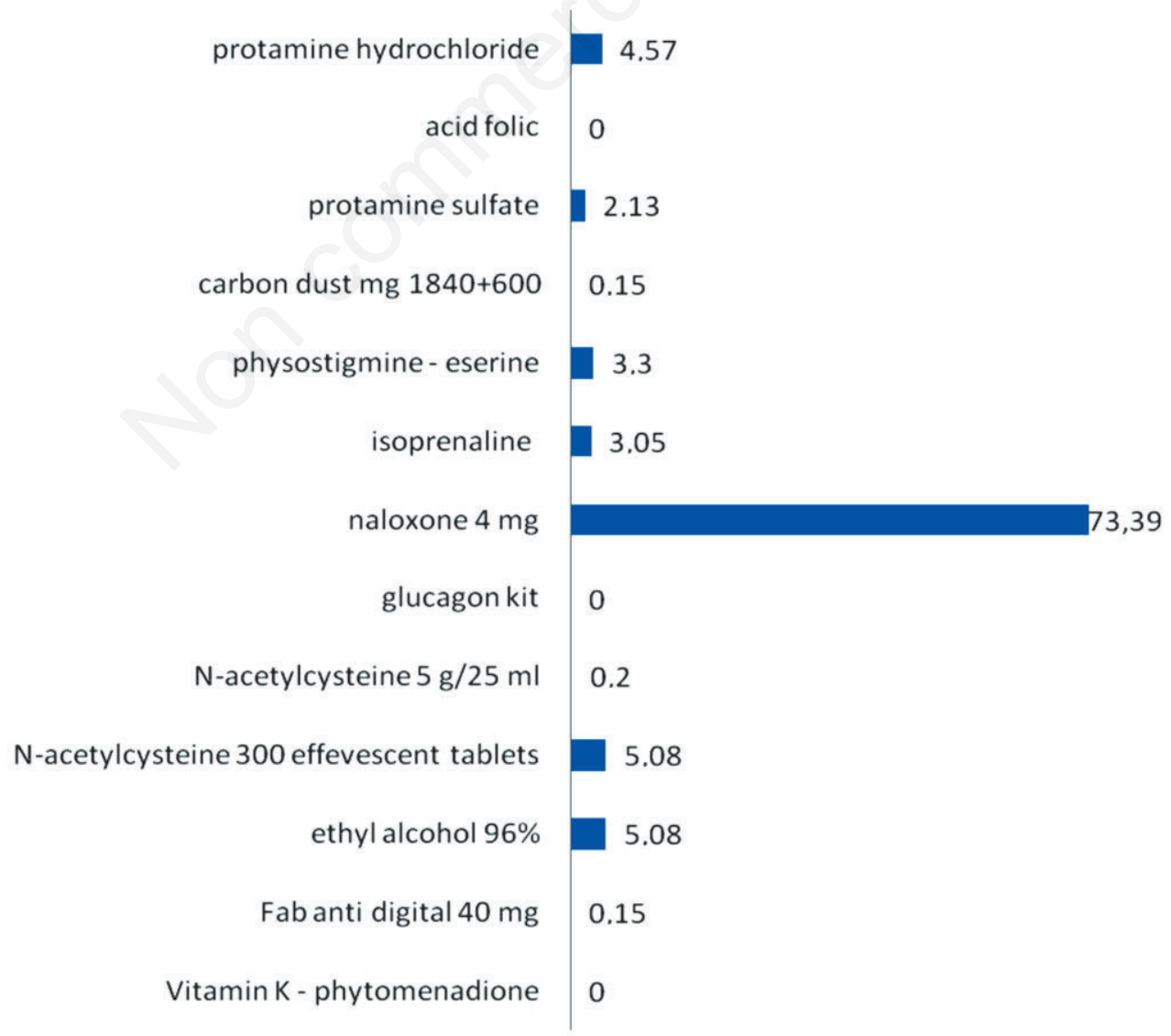

Figure 5. Percentage of antidotes used form 2009 to November 2012 at the Ospedali Riuniti Villa Sofia-Cervello, Palermo. 
Figure 6. Percentage of poisonings from 2009 to November 2012 at the three hospitals in the study.

some deficiencies in the stocks of hospital pharmacies. According to the comparison between the antidotes listed as being part of the pharmacy stock and their effective availability, and the list of national base data provided by the Pavia PCC, the lack of provision of antidotes could be explained in various ways. One could be inadequate knowledge or familiarity with antidotes on the part of doctors and pharmacists. Expensive antidotes that are used less and that have a short expiry date have to be paid for by the hospital and, therefore, in smaller and less important healthcare structures, the unavailability of antidotes is in part due to the lack of both economic and human resources.

One of the reasons is, of course, the high cost of some antidotes such as fomepizole that could be used instead of ethyl alcohol in methanol intoxications. In some other cases, quicker intervention could save lives, like the use of a hyperbaric chamber. Unfortunately, not all the hospitals have one due to their high maintenance costs, together with logistical problems and lack of space. Guidelines of the US Commission on Accreditation of Healthcare Organizations (JCAHO) show that the role of medical staff in the hospital and of the pharmacist in the hospital pharmacy is to determine which antidotes need to be available; the lack of suitable guidelines about minimum requirements for hospital supplies of antidotes can be one of the reasons for insufficient availability. Moreover, the results of this study indicate that specific toxicology training is one of the factors that could have a positive impact on adequate antidote availability. Generally speaking, specific training and provision of $a d$ hoc documentation could help respond to the problem of not having the appropriate antidotes available in an ER. Current recommendations more often refer to the appropriate antidote supply for special hospital services (e.g. Poison Control Centers) as well as other types of emergency services. As for the more rarely used and expensive antidotes, their availability across the Local Health Authority must be guaranteed according to specific criteria. These criteria must take into account the operative characteristics of each hospital and the local geographical characteristics. These must represent the basis for the creation of an availability network on a national level, even for those antidotes that are rarely used.

\section{References}

1. Botti P, Dannaoui B, Missanelli A, et al.; Gruppo di studio EPINTOX. Monitoraggio, valutazione clinica ed epidemiologica delle intossicazioni acute nei Pronto Soccorso e nei Dipartimenti di Emergenza. Firenze: Centro Antiveleni Firenze; 2004. Available from: http://www.antiveleni.altervista.org

2. Galli GC, Corsini E, Marinovich M. Tossicologia, 2nd ed. Padova: Piccin; 2008.

3. Zanutti M. Disponibilità e stoccaggio degli antidoti per l'emergenza ospedaliera. XXVII Congr. Sifo, 27-30 Settembre, Genova, Italy. [Abstract].

4. Locatelli C, Petrolini V, Lonati D, et al. Antidoti 2012, guida all'uso clinico e all'approvvigionamento. Pavia: Centro Antiveleni Pavia; 2012. Available from: http:// www.cavpavia.it

5. Lam SW, Engebretsen KM, Bauer SR. Toxicology today: what you need to know now. J Pharm Pract 2011;24:174-88. 